\title{
Hydrogen-rich saline alleviates early brain injury through regulating of ER stress and autophagy after experimental subarachnoid hemorrhage
}

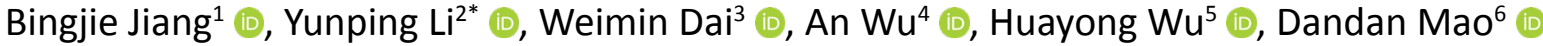

1. MM. Department of Neurosurgery - The Quzhou Affiliated Hospital - Wenzhou Medical University - Quzhou People's Hospital - Quzhou, China.

2. MM. Department of Neurosurgery - The Quzhou Affiliated Hospital - Wenzhou Medical University - Quzhou People's Hospital - Quzhou, China.

3. BS. Department of Neurosurgery - The Quzhou Affiliated Hospital - Wenzhou Medical University - Quzhou People's Hospital - Quzhou, China.

4. MM. Department of Neurosurgery - The Quzhou Affiliated Hospital - Wenzhou Medical University - Quzhou People's Hospital - Quzhou, China.

5. MM. Department of Neurosurgery - The Quzhou Affiliated Hospital - Wenzhou Medical University - Quzhou People's Hospital - Quzhou, China.

6. MM. Department of Neurosurgery - The Quzhou Affiliated Hospital - Wenzhou Medical University - Quzhou People's Hospital - Quzhou, China.

\begin{abstract}
Purpose: Subarachnoid hemorrhage $(\mathrm{SAH})$ is a common complication of cerebral vascular disease. Hydrogen has been reported to alleviate early brain injury (EBI) through oxidative stress injury, reactive oxygen species (ROS), and autophagy. Autophagy is a programmed cell death mechanism that plays a vital role in neuronal cell death after SAH. However, the precise role of autophagy in hydrogen-mediated neuroprotection following SAH has not been confirmed. Methods: In the present study, the objective was to investigate the neuroprotective effects and potential molecular mechanisms of hydrogen-rich saline in SAH-induced EBI by regulating neural autophagy in the C57BL/6 mice model. Mortality, neurological score, brain water content, ROS, malondialdehyde (MDA), and neuronal death were evaluated. Results: The results show that hydrogen-rich saline treatment markedly increased the survival rate and neurological score, increased neuron survival, downregulated the autophagy protein expression of Beclin-1 and LC3, and endoplasmic reticulum (ER) stress. That indicates that hydrogen-rich saline-mediated inhibition of autophagy and ER stress ameliorate neuronal death after SAH. The neuroprotective capacity of hydrogen-rich saline is partly dependent on the ROS/Nrf2/heme oxygenase-1 (HO-1) signaling pathway. Conclusion: The results of this study demonstrate that hydrogen-rich saline improves neurological outcomes in mice and reduces neuronal death by protecting against neural autophagy and ER stress.
\end{abstract}

Key words: Hydrogen. Brain Injuries. Oxidative Stress. Reactive Oxygen Species. Autophagy.

*Corresponding author: ypl019@163.com | (55 99) 9999-9999

Received: Apr 19, 2021 | Review: Jun 22, 2021 | Accepted: July 20, 2021

Conflict of interest: Nothing to declare.

Research performed at Department of Neurosurgery, The Quzhou Affiliated Hospital, Wenzhou Medical University, Quzhou People's Hospital, Quzhou, China. 


\section{Introduction}

Subarachnoid hemorrhage (SAH) is a common complication of cerebral vascular disease that is associated with a high rate of mortality, morbidity, and poor prognosis, especially in patients with hypertension. An occurrence of 6.2-10 per 100,000 has been recorded in Western countries $^{1-3}$. The key causes for SAH patients' poor outcomes were early brain damage (EBI) and cerebral vasospasm $(\mathrm{CVS})^{4}$. Recent clinical trials, however, have shown that drugs can greatly reduce CVS while having little impact on outcomes following $\mathrm{SAH}^{5}$, and previous clinical studies demonstrated it to $0^{6}$. The latest research has shown that $\mathrm{EBI}$ after SAH appears to play a critical role $\mathrm{e}^{7-10}$. The possible mechanisms underlying EBI include autophagy, apoptosis, direct neuronal death, and necroptosis ${ }^{8,11-13}$. However, Zille ${ }^{14}$ reported that inhibitors of caspase-dependent apoptosis, protein or mRNA synthesis, autophagy, mitophagy, or parthanatos had no effect in vitro or in vivo after intracerebral hemorrhage (ICH). Instead, inhibitors of ferroptosis defended against toxicity caused by hemoglobin and hemin. To date, it is unknown how often various types of cell death play a role in SAH-induced toxicity.

Under various physiological and pathological settings, autophagy is the primary cellular lysosomal degradation process for degrading and recycling intracellular proteins and organelles ${ }^{15}$. Autophagy has been shown to play a critical function in many central nervous system disorders, including traumatic brain injury (TBI) ${ }^{16-18}, \mathrm{ICH}^{19}, \mathrm{SAH}^{8}$, and Huntington's disease ${ }^{20}$. Tang ${ }^{17}$ found that inhibiting autophagy greatly reduce neuronal apoptosis and necrotic cell death, but the autophagy activator rapamycin can exacerbate brain injury. In turn, Fang ${ }^{18}$ stated that activating autophagy can reduce mitochondrial apoptosis, boost neurological function, cerebral edema, and relieve blood-brain barrier (BBB) disturbance after TBI in mice. Until nowadays, it was uncertain whether autophagy's neuroprotection was dependent on stimulation or inhibition. It was beneficial to investigate new possible drug targets focused on autophagy. Endoplasmic reticulum (ER) is the largest cellular organelle, in which all secreted and membrane proteins are synthesized and properly folded ${ }^{21}$. Previous studies had confirmed that ER stress play a vital important role in the early brain injury after $\mathrm{SAH}^{21-23}$.

Recently, hydrogen gas or hydrogen-rich saline have been commonly recognized for their ability to defend against a variety of diseases, including ischemia-reperfusion damage, stroke, spontaneous subarachnoid hemorrhage (SAH), and neurodegenerative diseases, by controlling oxidative stress, inflammatory response, and neuronal apoptosis ${ }^{24-27}$. Hydrogen has been shown in several experiments to selectively suppress hydroxyl radicals and peroxynitrites, and hence plays an important role in antioxidant, anti-apoptotic, anti-inflammatory, and cytoprotective properties ${ }^{24-28}$. However, the neuroprotective effects of hydrogen-rich saline therapy on SAH are debatable. Heme oxygenase- 1 (HO-1) is a cellular resistance enzyme that is caused by and protects against oxidant-induced damage. In the central nervous system, HO- 1 has anti-necroptosis, antineuroinflammatory, and neuroprotective effects (central nervous system - CNS) ${ }^{28,29}$. Previous study also confirmed that Nrf2/HO-1 can regulate neuron death in acute CNS disease $^{30}$. Thus, therapies targeting Nrf2 and HO-1 may be potential treatments for protection against inflammation, oxidative stress, and necroptosis after SAH. However, the exact mechanisms of the neuroprotective effects of hydrogenrich saline therapy remain unclear. It was investigated here the neuroprotective effect of hydrogen-rich saline therapy in a mice model of SAH through effects on neuroinflammation and necroptosis, and whether the neuroprotection was dependent on the ROS/Nrf2/HO-1 pathway.

\section{Methods}

The study protocol was approved by the Quzhou Affiliated Hospital of Wenzhou Medical University Research Ethics Committee (WYLL-2020-11). All animal experiments performed for this study complied with the National Institutes of Health guidelines for the handling of laboratory animals and were approved by the Ethics Committee of the Wenzhou Medical University. All experiments were conducted on healthy adult male C57BL/6J mice (8-10 weeks, 22-25 g) (Wenzhou Medical University, Wenzhou, China). Twenty-four mice were set in each group. The mice were housed in animal care facilities with a $12 \mathrm{~h}$ light/dark cycle and had free access to food and water.

\section{Animals SAH model}

The endovascular perforation method was used to construct the SAH model based on a protocol that was previously described ${ }^{7,31}$. Briefly, male C57BL6/J mice were anesthetized by intraperitoneal (i.p.) injection of $50-\mathrm{mg} / \mathrm{kg}$ pentobarbital sodium. Rectal temperature was kept at $37 \pm 0.5^{\circ} \mathrm{C}$ during operation using a heating pad. A midline incision was made in the neck, and left common external and internal carotid arteries were exposed. The left external carotid artery was ligated and cut, leaving a 3- $\mathrm{mm}$ stump, and a $4-0(0.33 \mathrm{~mm})$ monofilament nylon suture, $15 \mathrm{~mm}$ in length, was inserted into the left internal carotid artery through the external carotid artery stump to perforate the artery at the bifurcation of the anterior and middle cerebral artery. The suture was advanced $3 \mathrm{~mm}$ further to perforate the bifurcation of the anterior and middle cerebral arteries. After approximately $10 \mathrm{~s}$, the suture was withdrawn. Sham rats received similar surgical procedures, but without perforation. 


\section{Drug preparation and administration}

After the SAH model was established successfully, animals were given daily intraperitoneal injections of either hydrogen-rich $(5 \mathrm{~mL} / \mathrm{kg}$ ) (experimental) or plain (control) saline for 72 hours. The preparation of hydrogen-rich was according to the previous study ${ }^{32,33}$. Briefly, purified $\mathrm{H}_{2}$ was dissolved in normal saline for 2 hours under high pressure with 0.4 MPa, and the physiological concentration was kept at $1.73 \mathrm{~mL}$ hydrogen per $100 \mathrm{~mL}$ saline (average, more than $6 \mathrm{mmol} / \mathrm{L})$. Hydrogen-rich saline was stored at $4^{\circ} \mathrm{C}$ in an aluminum bag with no dead volume under atmospheric pressure. Hydrogen-rich saline was freshly prepared every week to ensure a constant concentration. The content of hydrogen in saline was evaluated and detected by gas chromatography, as a previous study reported ${ }^{34}$.

\section{Neurological function assessment}

The severity of early brain injury was evaluated by neurological function at 48 hours after $\mathrm{SAH}$ using a previously described neurological grading system ${ }^{7}$. The scoring system consisted of six tests, and specific standards are shown in Supplementary Table 1 . The neurological score, ranged from 3 to 18 , included spontaneous activities (0-3), movement symmetry of all limbs (0-3), forelimbs outstretching (0-3), body proprioception (1-3), response to vibrissae touch (1-3) and climbing (1-3). All rats from every group received a behavioral assessment, and a higher score represented a better neurological function.

\section{Mortality and SAH grade}

Mortality was documented 48 hours after SAH. SAH grade was given according to a previously described grading system ${ }^{35}$. Briefly, the grading was given based on subarachnoid blood blot:

- grade 0: no subarachnoid blood;

- grade 1: minimal subarachnoid blood;

- grade 2: moderate blood clot with appreciable arteries;

- grade 3: blood clot obliterating all arteries within the segment.

The grade ranges from 0 to 18 . Mice with $\mathrm{SAH}$ grading scores of less than 7, which had no prominent brain injury, were excluded from the study.

\section{Brain water content}

The severity of brain edema was evaluated by brain water content, which was determined by the standard wet-dry method as in previous studies ${ }^{7-10}$. The rats were sacrificed 48 hours after $\mathrm{SAH}$, and the entire brain was harvested and separated into the left and right cerebral hemispheres, followed by weighting cerebellum and brain stem (wet weight). Then, brain specimens from each group were dehydrated at $105^{\circ} \mathrm{C}$ for 24 hours to acquire the dry weight. The percentage of brain water content was equal to (wet weight - dry weight)/wet weight $\times 100 \%$.

\section{Evans blue extravasation}

Evans blue extravasation was performed as previously described $^{36}$. Briefly, mice were anesthetized by pentobarbital sodium ( $50 \mathrm{mg} / \mathrm{kg}$ ) injection 48 hours after $\mathrm{ICH} /$ obstructive sleep apnea (OSA). Evans blue dye (2\%, $5 \mathrm{~mL} / \mathrm{kg}$; SigmaAldrich, St. Louis, MO, United States) was injected into the left femoral vein over $2 \mathrm{~min}$ and circulated for $60 \mathrm{~min}$. Then, the mice were sacrificed with $100 \mathrm{mg} / \mathrm{kg}$ sodium pentobarbital via intraperitoneal injection and phosphatebuffered saline (PBS) intracardial perfusion. Death was clarified by observing respiration and by using the corneal reflection method. The brains were removed and quickly divided into the left and right cerebral hemispheres, weighed, homogenized in saline, and centrifuged at $15,000 \mathrm{~g}$ for $30 \mathrm{~min}$. Subsequently, the resultant supernatant was added with an equal volume of trichloroacetic acid, incubated overnight at $4^{\circ} \mathrm{C}$, and centrifuged at 15,000 g for $30 \mathrm{~min}$. Next, the resultant supernatant was collected and spectrophotometrically quantified at $610 \mathrm{~nm}$ for Evans blue dye.

\section{Analysis of reactive oxygen species}

The non-fluorescent diacetylated 2',7'-dichlorofluorescein (DCF-DA) probe (Sigma-Aldrich, St. Louis, MO, United States), which becomes highly fluorescent upon oxidation, was used to evaluate intracellular ROS production according to the manufacturer's instructions ${ }^{37}$.

\section{Analysis of lipid peroxidation}

Malondialdehyde (MDA) levels were detected by lipid peroxidation assay kit (Ex/Em 532/553 nm, Ab118970, Abcam, Cambridge, United Kingdom), according to the manufacturer's instructions ${ }^{38}$.

\section{TUNEL staining}

A terminal deoxynucleotidyl transferase dUTP nick end labeling (TUNEL) assay was conducted to assess neuronal death in the brain cortex according to the previous study ${ }^{31}$. TUNEL reaction mixture $(50 \mu \mathrm{L})$ was added to each sample, and the slides were incubated in a humidified dark chamber for $60 \mathrm{~min}$ at $37^{\circ} \mathrm{C}$. The slides were then incubated with DAPI for 5 minutes at room temperature in the dark to stain the nuclei, followed by imaging with a fluorescence microscope. The procedure was performed according to the manufacturer's instructions with a TUNEL staining kit. A negative control (without the TUNEL reaction mixture) was used. The apoptotic index (\%) was the ratio of the 
number of TUNEL-positive cells/total number of cells $\times 100$. The cell count was confirmed in four randomly selected high-power fields, and the data obtained from each field were averaged.

\section{Western blot analysis}

Western blot analysis was performed as indicated previously ${ }^{39}$. Briefly, cerebral cortex or hippocampus samples were collected, dissolved, and separated by sodium dodecyl sulfate-polyacrylamide gel electrophoresis in $10 \%$ polyacrylamide gels. A BCA protein assay kit (Beyotime) was used to measure protein concentrations by the bicinchoninic acid method. Then, protein samples were transferred onto immobilon nitrocellulose membranes. The membranes were blocked at room temperature for $1 \mathrm{~h}$ with $5 \%$ nonfat milk.

The membranes were then incubated with the following primary antibodies overnight at $4^{\circ} \mathrm{C}$ :

- rabbit anti- $\beta$-actin (1:1,000, Abcam, ab8227);

- rabbit CHOP (\#5554, Cell Signaling, 1:1,000);

- rabbit anti-cleaved-caspase-12 (\#2202, Cell Signaling, 1:200);

- rabbit anti-GRP78 (\#3183, Cell Signaling, 1:800);

- rabbit anti-Beclin-1 (1 $\mu \mathrm{g} / \mathrm{mL}$, Abcam, ab62557);

- rabbit anti-Nrf2 (1:1,000, rabbit polyclonal, Abcam, ab31163);

- rabbit anti-HO-1 (1:1,000, rabbit polyclonal, Abcam, ab13243);

- rabbit anti-LC-3B (1 $\mu \mathrm{g} / \mathrm{mL}$, rabbit monoclonal, Abcam, ab48394).

After washing the membranes with TBST three times, HRP-conjugated goat anti-rabbit IgG or goat anti-mouse IgG secondary antibodies $(1: 5,000)$ were applied, and the membranes were incubated in the secondary antibodies at room temperature for $1.5 \mathrm{~h}$. The protein bands were detected using a Bio-Rad imaging system (Bio-Rad, Hercules, CA, United States) and quantified with ImageJ.

\section{Statistical analysis}

All experiments were repeated more than three times, and the data are expressed as the means and scanning electron microscope (SEM). Statistical Package for the Social Sciences 14.0 (SPSS, Chicago, IL, United States) and GraphPad Prism 6 (GraphPad Software, San Diego, CA, United States) were used for the statistical analyses. Student's t-test was used when two groups were compared, and one-way analysis of variance (ANOVA) followed by Bonferroni's post-hoc test was used for the comparison of two independent variables. For non-normally distributed data and/or non-homogeneous variance, Kruskal-Wallis test was used followed by Dunn's post-hoc test. For all the statistical analyses, data were considered significant at $\mathrm{p}<0.05$.

\section{Results}

\section{Treatment with hydrogen-rich saline has no long- term effects neither on mortality rates nor on SAH grade in SAH models}

To clarify the neuroprotection of hydrogen-rich saline, the endovascular perforation method was used to construct the SAH model in vivo. The effect of hydrogen-rich saline treatment on the neurological damage parameters was evaluated, including mortality rates and $\mathrm{SAH}$ grades. As shown in Fig. 1, mortality rates (Fig. 1a) and SAH grades (Fig. 1b) in various groups, including sham, $\mathrm{SAH}, \mathrm{SAH}+$ hydrogen-rich saline ( $\mathrm{SAH}+\mathrm{HS}$ ) did not significantly differ, suggesting that hydrogen-rich saline treatment has no effects in alleviating $\mathrm{SAH}$ in long term. So, the focus was on assessing the value of hydrogen-rich saline treatment on early brain injury in the following studies.
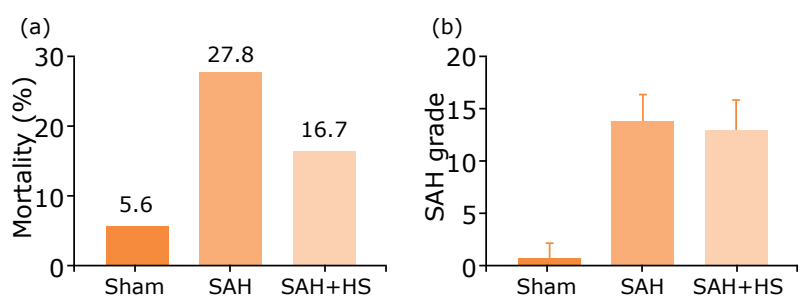

SAH: Subarachnoid hemorrhage; HS: hydrogen-rich saline.

Figure 1 - Treatment with hydrogen-rich saline has no long-term effects neither on mortality rates nor on $\mathrm{SAH}$ grade in SAH models. (a) Mortality rates in the sham group (5.6\%), SAH group (27.8\%), and the SAH + HS group (16.7\%). No significant differences between the three groups. (b) SAH grade scores in the sham group, the SAH group, and the SAH + HS group, which showed no significant differences (one-way analysis of variance [ANOVA]).

\section{Hydrogen-rich saline alleviates EBI after SAH}

To clarify the neuroprotection of hydrogen-rich saline after SAH, modified neurological severity scores were used to evaluate neurological deficits, and brain water content by the wet-dry and Evans blue extravasation method at $48 \mathrm{~h}$ after SAH to evaluate brain damage.

The results showed that $\mathrm{SAH}$ increased the brain water content significantly ( $p<0.05$, Fig. 2a), and BBB permeability ( $p<0.05$, Fig. $2 b)$, which was alleviated after hydrogen-rich saline treatment. Similar results were found in neurological scores, which were decreased significantly after $\mathrm{SAH}$, and hydrogen-rich saline induction can significantly improve the neurological function ( $p<0.05$, Fig. $2 c$ ). Neuronal damage and death were the main reason that leads to $\mathrm{EBI}$ after $\mathrm{SAH}$. So, TUNEL assay was used to evaluate the level of 
cell death in treated and untreated with hydrogen-rich saline in the SAH mice at $48 \mathrm{~h}$ after model construction. The hippocampus neuronal death decreased after hydrogenrich saline treatment (Fig. 2d). These results demonstrate that hydrogen-rich saline has neuroprotective effects after $\mathrm{SAH}$.
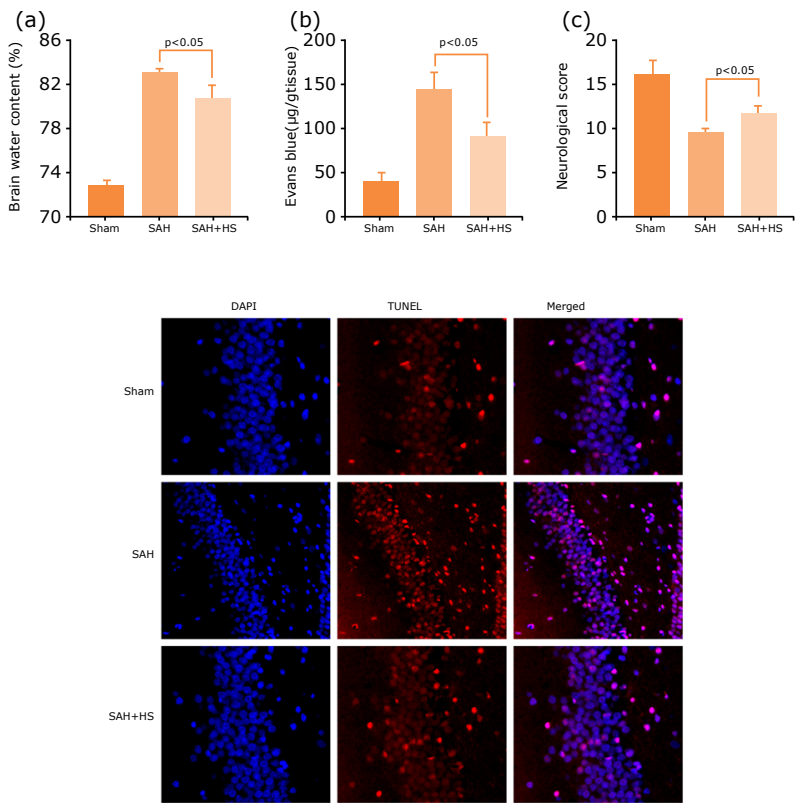

SAH: subarachnoid hemorrhage; HS: hydrogen-rich saline; TUNEL: terminal deoxynucleotidyl transferase dUTP nick end labeling; EBI: early brain injury; BBB: blood-brain barrier; ANOVA: analysis of variance; SEM: scanning electron microscope.

Figure 2 - Hydrogen-rich saline alleviates EBI after SAH. (a) Hydrogen-rich saline alleviates brain water content significantly after SAH $(n=6, p<0.05)$. (b) Hydrogenrich saline alleviates BBB permeability after SAH $(n=6$, $p<0.05)$. (c) Neurological score of mice in the sham group, $\mathrm{SAH}$ group and $\mathrm{SAH}+\mathrm{HS}$ group at $48 \mathrm{~h}$, hydrogenrich saline increased the neurological score significantly $(n=10, p<0.05)$. (d) TUNEL assay showed that hydrogenrich saline alleviates neuronal death. $p<0.05$; ANOVA; mean \pm SEM.

\section{Hydrogen-rich saline inhibited SAH-induced autophagy activation in the hippocampus}

To clarify whether autophagy plays an important role in SAH and hydrogen-rich saline can regulate autophagy, the expression levels of autophagy-related protein by western blotting were also detected (Fig. 3a). The results of western blotting indicated that hydrogen-rich saline can reduce the expression levels of autophagy-related protein Beclin-1 and LC3 (Fig. 3b-c). The immunofluorescent staining showed that LC3-positive neurons were hardly observed in the hippocampus, widespread among the hippocampus after SAH induction, but they decreased after hydrogenrich saline administration (Fig. 3d). (a)

(b)
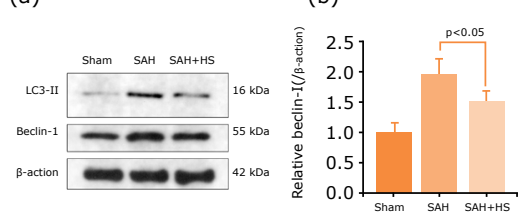

(c)

(d)

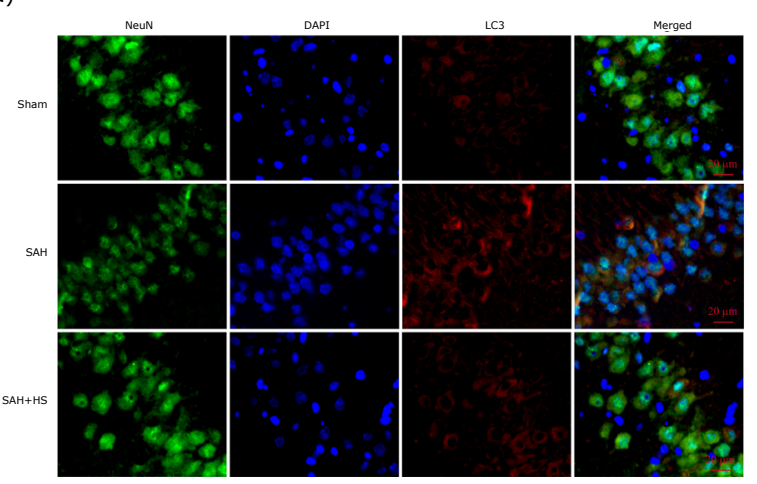

SAH: subarachnoid hemorrhage; HS: hydrogen-rich saline;

ANOVA: analysis of variance; SEM: scanning electron microscope. Figure 3 - Hydrogen-rich saline inhibited SAH-induced autophagy activation in the hippocampus. (a) Expression of autophagy-related proteins, Beclin-1 and LC3 in the hippocampus of mice after $\mathrm{SAH}$ were determined by Western blotting. (b-c) Quantification of Beclin-1 and LC3 protein levels in the hippocampus to actin loading control, hydrogen-rich saline decreased Beclin-1 and LC3 expression after SAH in mice. (d) Immunocytochemistry shows that hydrogen-rich saline downregulated LC3 expression in the hippocampus. $n=6 ; p<0.05$; ANOVA; mean \pm SEM.

\section{Hydrogen-rich saline alleviates ER stress}

To investigate the effect of hydrogen-rich saline on ER stress after SAH, the ER stress core markers were GRP78, CHOP, and caspase-12. We detected the expression of ER stress-associated proteins by Western blot (Fig. 4a). The results of Western blot also indicated that hydrogen-rich saline can reduce the expression levels of ER stress-related protein GRP78, CHOP, and caspase-12 (Fig. 4b-d). Hence, it is supposed that the neuroprotection of hydrogen-rich saline is partly based on ER stress inhibition.

\section{Rapamycin stimulates autophagy and reversed the neuroprotective effect of hydrogen-rich saline}

Rapamycin was a specific activator for autophagy ${ }^{39}$. To investigate the relationship between autophagy and the neuroprotective role of hydrogen-rich saline, mice were pretreated with rapamycin before the induction of SAH. The results showed that pretreated with rapamycin could dramatically damage neurological deficits (Fig. 5a), aggravate brain edema (Fig. 5b) and BBB permeability (Fig. 5c), and reverse the neuroprotective effect of hydrogen-rich saline. 
(a)

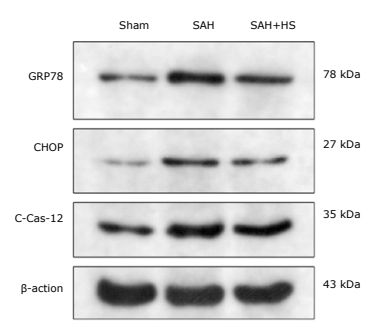

(c)

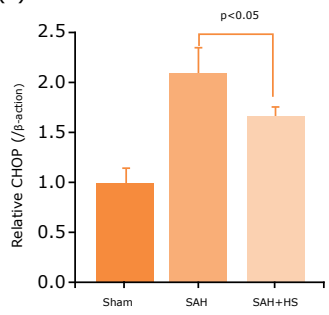

(b)

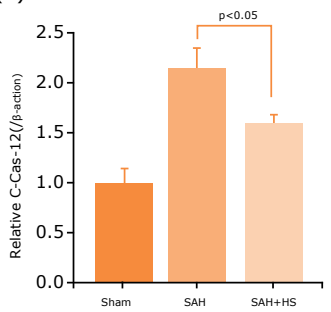

(d)

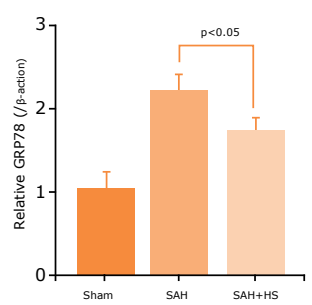

SAH: subarachnoid hemorrhage; HS: hydrogen-rich saline; C-Cas-12: caspase-12; ER: endoplasmic reticulum stress; ANOVA: analysis of variance; SEM: scanning electron microscope.

Figure 4 - Hydrogen-rich saline alleviates ER stress. (a) Expression of ER stress-related proteins, caspase-12, CHOP and GRP78 in the cerebral cortex of mice after $\mathrm{SAH}$ were determined by Western blotting. (b-d) Quantification of caspase-12, CHOP and GRP78 protein levels in the cerebral cortex to actin loading control, hydrogen-rich saline decreased caspase-12, CHOP and GRP78 expression after $\mathrm{SAH}$ in mice. $n=6 ; p<0.05$; ANOVA; mean \pm SEM.

Additionally, the TUNEL assay also showed that rapamycin could also significantly increase the neuron apoptosis in the injured hippocampus, compared with the $\mathrm{SAH}+$ hydrogen-rich saline group (Fig. 5d). The autophagyrelated protein expression by Western blot was detected too (Fig. 5e). Hydrogen-rich saline can significantly decrease the expression levels of Beclin-1, and LC3 (Fig. 5f), while partly blocked with rapamycin administration. Thus, these results indicated that rapamycin could activate autophagy and abolish the anti-autophagy effects of hydrogen-rich saline, then reversed the neuroprotective effects of hydrogen-rich saline after SAH.

\section{Hydrogen-rich saline regulates autophagy and $E R$} stress by ROS/Nrf2/HO-1 signaling pathway after SAH

Autophagy through ROS/Nrf2/HO-1 signaling pathway after hydrogen-rich treatment was explored. It was detected the ROS levels by DCF-DA probe, and the degree of membrane lipid peroxidation was evaluated by MDA. The results showed that ROS and MDA levels were significantly increased after $\mathrm{SAH}$, while they decreased after hydrogenrich treatment (Fig. 6a-b). The protein expression levels

of Nrf2 and HO-1 by Western blot to investigate neuron autophagy were also detected (Fig. 6c). The results showed that the expression levels of Nrf2 and HO-1 decreased significantly in the SAH group, and increased after hydrogenrich saline administration (Fig. 6d-e). Thus, these results showed that hydrogen-rich saline may have inhibited $\mathrm{SAH}$-induced autophagy by a regulated ROS/Nrf2/HO-1 signaling pathway. (a)

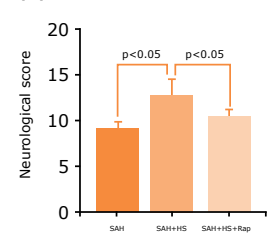

(b)

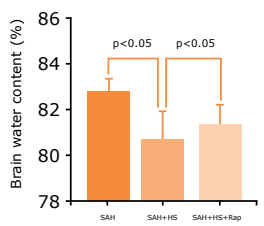

(c)

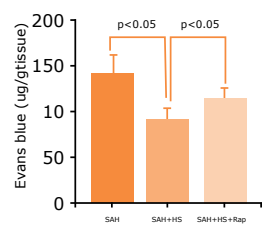

(d)
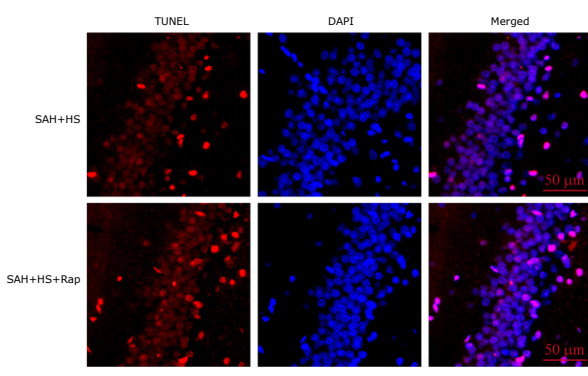

(e)

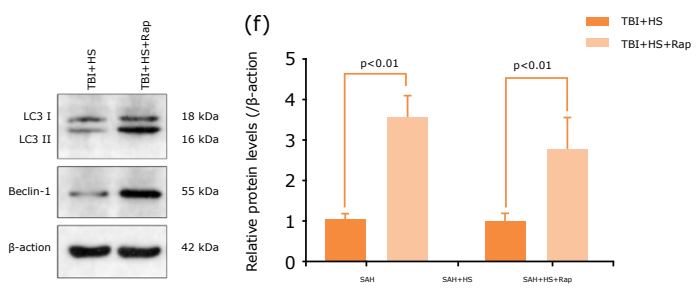

SAH: subarachnoid hemorrhage; HS: hydrogen-rich saline; Rap: rapamycin; BBB: blood-brain barrier; TUNEL: terminal deoxynucleotidyl transferase dUTP nick end labeling; ANOVA: analysis of variance; SEM: scanning electron microscope.

Figure $\mathbf{5}$ - Rapamycin stimulates autophagy and reversed the neuroprotective effect of hydrogen-rich saline. (a) Hydrogen-rich saline increased the neurological score $(n=6, p<0.05)$. (b) Hydrogen-rich saline alleviated brain water content significantly after $\mathrm{SAH}$, while aggravated it after rapamycin administration $(n=6, p<0.05)$. (c) Hydrogen-rich saline alleviated BBB permeability after $\mathrm{SAH}$, while aggravated it after rapamycin administration ( $n=6, p<0.05)$. (d) TUNEL assay showed that autophagy activator increased neuronal death, and reversed the neuroprotective effect of hydrogen-rich saline. (e) Expression of autophagy-related proteins, LC3 and Beclin-1 after SAH were determined by Western blotting. (f) Rapamycin increased the expression levels of LC3 and Beclin-1 significantly than the $\mathrm{SAH}+\mathrm{HS}$ group $(\mathrm{n}=6$, $p<0.05) . n=6 ; p<0.05$; ANOVA; mean \pm SEM. 
(a)

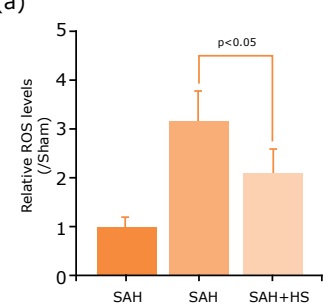

(a)

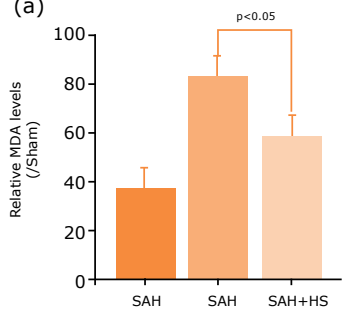

(c)
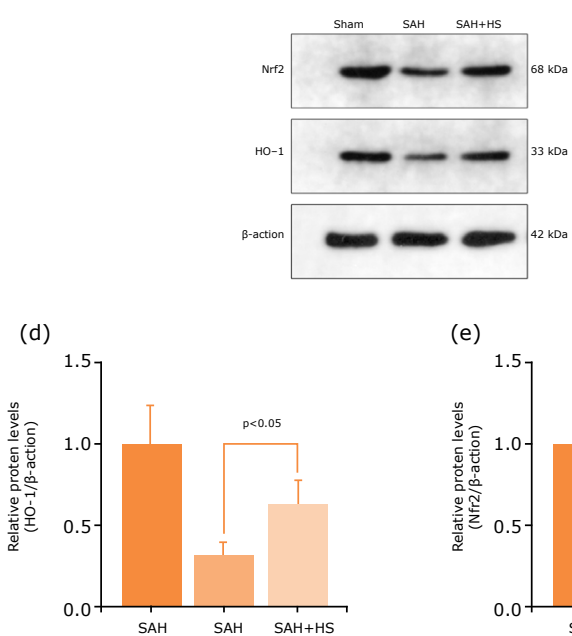

(e)

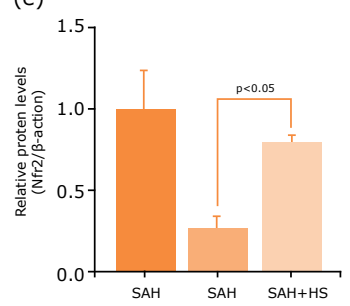

SAH: subarachnoid hemorrhage; HS: hydrogen-rich saline; ROS: reactive oxygen species; MDA: malondialdehyde; HO-1: heme oxygenase-1; ER: endoplasmic reticulum stress; ANOVA: analysis of variance; SEM: scanning electron microscope.

Figure 6 - Hydrogen-rich saline regulated autophagy and ER stress by ROS/Nrf2/HO-1 signaling pathway after SAH. (a) Hydrogen-rich saline decreased ROS levels after SAH by DCF-DA probe. (b) Hydrogen-rich saline decreased MDA levels after SAH. (c) Expression of autophagy-related proteins, Nrf2 and HO-1 after SAH were determined by Western blotting. (d-e) Nrf2 and HO-1 protein levels were quantificated in the cerebral cortex to actin loading control, hydrogen-rich saline increased Nrf2 and HO-1 expression after SAH in mice. $n=6 ; p<0.05$; ANOVA; mean \pm SEM.

\section{Discussion}

Here, the therapeutic potential of hydrogen-rich saline for alleviating early brain injury in a mouse in the SAH model was evaluated. The present study demonstrates that hydrogenrich saline was a neuroprotective agent that can attenuate $\mathrm{EB}$ following $\mathrm{SAH}$. It was found that hydrogen-rich saline can improve neurological dysfunction after $\mathrm{SAH}$; hydrogen-rich saline can alleviate brain damage in a mouse $\mathrm{SAH}$ model; hydrogen-rich saline can relieve ER stress after $\mathrm{SAH}$; hydrogen-rich saline can prevent autophagy after ER stress and alleviate neuronal death; and the anti-ER stress and anti-autophagy roles of hydrogen-rich saline may be related to the ROS/Nrf2/HO-1 pathway (Fig. 7).

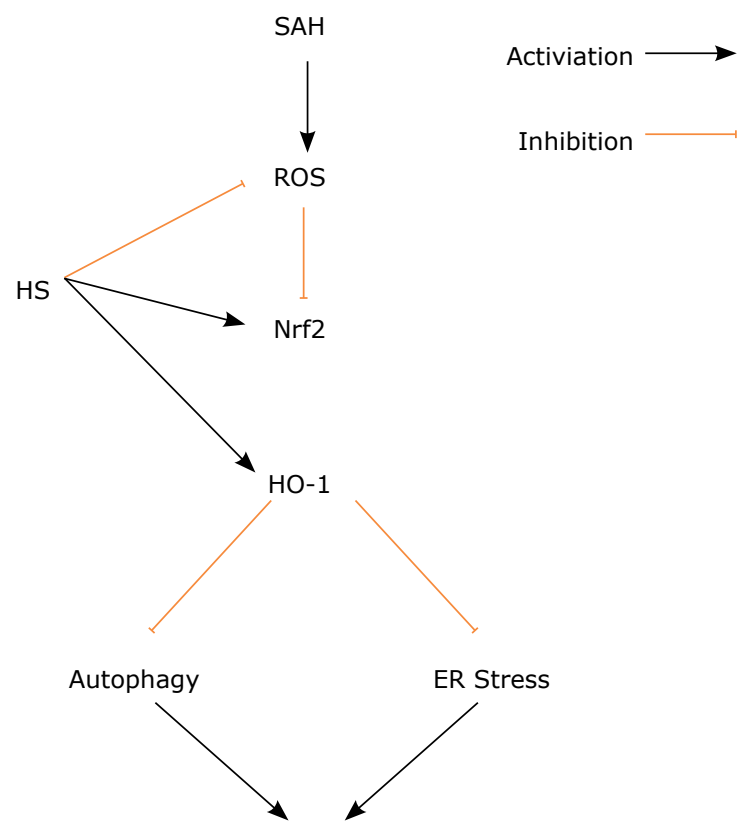

EBI Aggtavated

SAH: subarachnoid hemorrhage; HS: hydrogen-rich saline; ROS: reactive oxygen species; HO-1: heme oxygenase-1; ER: endoplasmic reticulum stress; $\mathrm{EBI}$ : early brain injury.

Figure 7 - Diagram of the proposed model explaining the observations of autophagy and ER stress after SAH and potential mechanisms underlying the effect of the hydrogen-rich saline intervention.

Hydrogen gas or hydrogen-rich saline can easily penetrate the BBB by gaseous diffusion, which is widely accepted to exert protective effects in many CNS diseases, including ischemia stroke, intracranial hemorrhage, $\mathrm{TBI}$, and neurodegenerative diseases ${ }^{24-27}$. Hydrogen gas or hydrogenrich saline plays an important role in antioxidant activity with high tissue transferability, and previous studies had demonstrated that $\mathrm{H}_{2}$ is safe for patients and animals ${ }^{34}$. The anti-oxidative stress and anti-inflammatory response of hydrogen gas or hydrogen-rich saline are induced by selective inhibition of highly toxic ROS, such as hydroxyl radical $(\mathrm{OH} \cdot)$ and peroxynitrite (ONOO- $)^{26}$.

$\mathrm{Liu}^{40}$ reported that $\mathrm{H}_{2}$ can markedly improve the survival rate and cognitive dysfunction, decrease inflammatory response and oxidative stress, and increase activities of antioxidant enzymes in serum and hippocampus in a mouse model of sepsis. In the ICH model, it was also found that hydrogen plays a neuroprotective effect against EBI after $\mathrm{ICH}$, alleviating brain edema and neurologic deficits through regulating oxidative stress, neuroinflammation, and apoptosis ${ }^{41}$.

In the hypoxic-ischemic brain injury neonatal rats' model, $\mathrm{H}_{2}$ inhalation administration can alleviate brain 
damage and improve early neurological outcomes, the mechanisms also through antioxidant, antiapoptotic, and anti-inflammatory responses via MAPK/HO-1/PGC-1a pathway ${ }^{42}$. In the TBI model, molecular hydrogen water also can reverse the controlled cortical impact-induced brain edema through the preservation or increase of adenosine triphosphate (ATP) levels ${ }^{43}$. A pilot rats study indicated that high-dose hydrogen gas therapy reduces mortality and improves outcomes after $\mathrm{SAH}^{44}$.

Zhuang reported that hydrogen can alleviate brain injury via decreasing oxidative stress injury and brain edema in experimental SAH rabbits ${ }^{32}$. Hydrogen-rich saline can improve neurological function, decrease neuronal apoptosis by upregulating $\mathrm{Bcl}-2$ and downregulate Bax and cleave caspase-3 after SAH. The potential mechanism may be through Akt/GSK3 $\beta$ signaling pathway. In the present study, we also found that hydrogen-rich saline markedly increased the survival rate and neurological score, alleviated brain edema, and increased neuron survival.

Autophagy regulates the turnover of cellular constituents to ensure the removal and recycling of toxins and was very important in cell homeostasis. The role of autophagy has been confirmed in many CNS diseases, including acute brain injury ${ }^{16-18}, \mathrm{ICH}^{19}, \mathrm{SAH}^{39}$, and Huntington's disease ${ }^{20}$. Autophagy can transport materials in cells to lysosomes for degradation through different pathways, involved in the regulation of cell survival and death mechanisms after $\mathrm{SAH}$.

Therefore, autophagy plays a very important role in neuronal injury and repair after $\mathrm{SAH}$. In the myocardial ischemia/reperfusion (I/R) in vitro and in vivo model, hydrogen-rich saline can improve the inflammatory response and apoptosis via PINK1/Parkin mediated autophagy ${ }^{45}$. Chen ${ }^{46}$ reported that $\mathrm{H}_{2}$ can alleviate vital organ damage, inhibited lipopolysaccharide (LPS) and ATP caused by NLRP3 inflammasome pathway activation, and improve mitochondrial dysfunction via regulating autophagy. Recent studies also indicated that hydrogen-rich saline or hydrogen gas can decrease cell death via regulating autophagy ${ }^{47-50}$. So far, this is the first report that hydrogen-rich saline can alleviate EBI after SAH by regulating autophagy. In the present study, it was found that autophagy was excessive activated after $\mathrm{SAH}$, then led to neurologic impairment, BBB disruption, brain edema, and neuronal death, while it was reversed after hydrogen-rich saline treatment.

The molecular mechanism of autophagy and ER stress is complicated, and the exact mechanisms of the neuroprotective effects of hydrogen-rich saline therapy remain unclear. Nrf2 was a very important transcriptional regulation factor that can regulate the expression of more than 250 genes and is marked by its binding site, antioxidant response element, most genes can regulate oxidative stress and cell apoptosis, necroptosis, autophagy, and ferroptosis ${ }^{30}$.

$\mathrm{Yu}^{51}$ reported that $2 \%$ molecular hydrogen $\left(\mathrm{H}_{2}\right)$ gas inhalation can improve the survival rates, reduce the lung edema and the lung injury score, and ameliorate the injuries caused by oxidative stress and inflammation in the septic mice model. Knockout Nrf2 would reverse or weaken the protection of $\mathrm{H}_{2}$ gas on lung damage, and also depends on the $\mathrm{HO}-1$ and high-mobility group box 1 (HMGB1).

Additionally, Chen ${ }^{52}$ demonstrated that $\mathrm{H}_{2}$ attenuates endothelial injury and inflammation, increased the HO-1 expression and in-vitro and in-vivo activity, and knockout Nrf2 or HO-1 inhibition reversed the protection of $\mathrm{H}_{2}$, the process depending on the activity of $\mathrm{Nrf2} / \mathrm{HO}-1$ signaling pathway. $\mathrm{Yu}^{53}$ reported that $\mathrm{H}_{2}$ can improve survival in septic mice, and decrease escape latency and platform crossing times, the brain water content, and extravascular dextran, while reversed in the Nrf2 knockout mice. Wang ${ }^{42}$ pointed out that hydrogen gas can alleviate hypoxic-ischemic EBI via regulating the HO-1 pathway. Intriguingly, the present study found that knockdown $\mathrm{HO}-1$ reversed the neuroprotection of hydrogen-rich saline after $\mathrm{SAH}$, and $\mathrm{HO}-1$ might be the upstream signal of ER stress and autophagy. However, the exact mechanism needs to be further determined.

\section{Conclusions}

The present study provided evidence that autophagy, which is mediated by the ROS/Nrf2/HO-1, is an important cellular regulatory mechanism and contributes to $\mathrm{EBI}$ after $\mathrm{SAH}$. In this study, for the first time, it was reported that hydrogen-rich saline induced regulation of autophagy and ER stress, and also a new idea was provided to explore the biological effects and underlying mechanisms of the hydrogen-rich saline.

\section{Author's contribution}

Conception of the study: Jiang $B, W u ~ H$ and Mao D; Design the study: Jiang B, Li Y, Dai W and Wu A; Acquisition of data: Jiang B and Li Y; Manuscript writing: Jiang B, Li Y and Mao D; Critical revision: Jiang B, Li Y and Mao D; Final approval: Jiang B, Li Y, Dai W, Wu A, Wu H and Mao D.

\section{Data availability statement}

Data will be available upon request.

\section{Funding}

115 talents of Quzhou

Grant No: 2019 


\section{Acknowledgments}

Not applicable.

\section{Reference:}

1. Etminan N, Chang H-S, Hackenberg K, de Rooij NK, Vergouwen MDI, Rinkel GJE, Algra A. Worldwide incidence of aneurysmal subarachnoid hemorrhage according to region, time period, blood pressure, and smoking prevalence in the population a systematic review and meta-analysis. Jama Neurol. 2019;76(5):588-97. https:// doi.org/10.1001/jamaneurol.2019.0006

2. Korja $M$, Lehto $H$, Juvela $S$, Kaprio J. Incidence of subarachnoid hemorrhage is decreasing together with decreasing smoking rates. Neurology. 2016;87(11):111823. https://doi.org/10.1212/wnl.0000000000003091

3. Mackey J, Khoury JC, Alwell K, Moomaw CJ, Kissela BM, Flaherty ML, Adeoye O, Woo D, Ferioli S, La Rosa FDLR, Martini S, Khatri P, Broderick JP, Zuccarello M, Kleindorfer D. Stable incidence but declining case-fatality rates of subarachnoid hemorrhage in a population. Neurology. 2016;87(21):2192-7. https://doi.org/10.1212/ wnl.0000000000003353

4. Chen J, Zhu J, He J, Wang Y, Chen L, Zhang C, Zhou J, Yang L. Ultra-early microsurgical treatment within $24 \mathrm{~h}$ of SAH improves prognosis of poor-grade aneurysm combined with intracerebral hematoma. Oncol Lett. 2016;11(5):31738. https://doi.org/10.3892/ol.2016.4327

5. Macdonald RL, Higashida RT, Keller E, Mayer SA, Molyneux A, Raabe A, Vajkoczy P, Wanke I, Bach D, Frey A, Marr A, Roux S, Kassell N. Clazosentan, an endothelin receptor antagonist, in patients with aneurysmal subarachnoid haemorrhage undergoing surgical clipping: a randomised, double-blind, placebo-controlled phase 3 trial (CONSCIOUS-2). Lancet Neurol. 2011;10(7):618-25. https://doi.org/10.1016/s1474-4422(11)70108-9

6. Chen J, Li M, Zhu X, Chen L, Yang S, Zhang C, Wu T, Feng X, Wang $Y, C$ Chen $Q$. Atorvastatin reduces cerebral vasospasm and infarction after aneurysmal subarachnoid hemorrhage in elderly Chinese adults. Aging (Albany NY). 2020;12(3):293951. https://doi.org/10.18632/aging.102788.

7. Chen J, Xuan Y, Chen Y, Wu T, Chen L, Guan H, Yang S, He J, Shi $D$, Wang Y. Netrin-1 alleviates subarachnoid haemorrhageinduced brain injury via the PPAR gamma/NF-KB signalling pathway. J Cell Mol Med. 2019;23(3):2256-62. https://doi. org/10.1111/jcmm.14105

8. Chen J-H, Wu T, Xia W-Y, Shi Z-H, Zhang C-L, Chen L, Chen Q-X, Wang $Y-H$. An early neuroprotective effect of atorvastatin against subarachnoid hemorrhage. Neural Regen Res. 2020;15(10):194754. https://doi.org/10.4103/1673-5374.280326

9. Chen J-H, Wu T, Yang L-K, Chen L, Zhu J, Li P-P, Hu X, Wang $\mathrm{Y}-\mathrm{H}$. Protective effects of atorvastatin on cerebral vessel autoregulation in an experimental rabbit model of subarachnoid hemorrhage. Mol Med Rep. 2018;17(1):16519. https://doi.org/10.3892/mmr.2017.8074
10. Chen J-H, Yang L-K, Chen L, Wang $\mathrm{Y}-\mathrm{H}, \mathrm{Wu} \mathrm{Y}$, Jiang $\mathrm{B}-\mathrm{J}$, Zhu J, Li P-P. Atorvastatin ameliorates early brain injury after subarachnoid hemorrhage via inhibition of AQP4 expression in rabbits. Int J Mol Med. 2016;37(4):1059-66. https://doi.org/10.3892/ijmm.2016.2506

11. Cahill J, Zhang JH. Subarachnoid hemorrhage is it time for a new direction? Stroke. 2009;40(3):S86-S7. https://doi. org/10.1161/strokeaha.108.533315

12. Dong Y, Fan C, Hu W, Jiang S, Ma Z, Yan X, Deng C, Di S, Xin Z, Wu $G$, Yang Y, Reiter RJ, Liang G. Melatonin attenuated early brain injury induced by subarachnoid hemorrhage via regulating NLRP3 inflammasome and apoptosis signaling. J Pineal Res. 2016;60(3):253-62. https://doi.org/10.1111/jpi.12300

13. Kenny EM, Fidan E, Yang $Q$, Anthonymuthu TS, New LA, Meyer EA, Wang $\mathrm{H}$, Kochanek PM, Dixon CE, Kagan VE, Bayir $\mathrm{H}$. Ferroptosis contributes to neuronal death and functional outcome after traumatic brain injury. Crit Care Med. 2019;47(3):410-8. https://doi.org/10.1097/ ccm.0000000000003555

14. Zille M, Karuppagounder SS, Chen YX, Gough PJ, Bertin J, Finger J, Milner TA, Jonas EA, Ratan RR. Neuronal death after hemorrhagic stroke in vitro and in vivo shares features of ferroptosis and necroptosis. Stroke. 2017;48(4):103343. https://doi.org/10.1161/strokeaha.116.015609

15. Ceccariglia S, Cargnoni A, Silini AR, Parolini O. Autophagy: a potential key contributor to the therapeutic action of mesenchymal stem cells. Autophagy. 2020;16(1):28-37. https://doi.org/10.1080/15548627.2019.1630223

16. Li H, Lu C, Yao W, Xu L, Zhou J, Zheng B. Dexmedetomidine inhibits inflammatory response and autophagy through the circLrp1b/miR-27a-3p/Dram2 pathway in a rat model of traumatic brain injury. Aging (Albany NY). 2020;12(21):21687705. https://doi.org/10.18632/aging.103975

17. Tang $C$, Shan $Y$, Hu $Y$, Fang $Z$, Tong $Y$, Chen $M$, Wei $X, F u X, X u X$. FGF2 Attenuates neural cell death via suppressing autophagy after rat mild traumatic brain injury. Stem Cells Int. 2017;2017:2923182. https://doi. org/10.1155/2017/2923182

18. Fang J, Zhu Y, Wang $H$, Cao B, Fei M, Niu W, Zhou Y, Wang X, Li X, Zhou M. Baicalin protects mice brain from apoptosis in traumatic brain injury model through activation of autophagy. Front Neurosci. 2018;12:1006. https://doi. org/10.3389/fnins.2018.01006

19. Zhao M, Gao J, Cui C, Zhang Y, Jiang X, Cui J. Inhibition of PTEN ameliorates secondary hippocampal injury and cognitive deficits after intracerebral hemorrhage: involvement of AKT/FoxO3a/ATG-mediated autophagy. Oxid Med Cell Longev. 2021;2021:5472605. https://doi. org/10.1155/2021/5472605

20. Aron R, Pellegrini P, Green EW, Maddison DC, Opoku-Nsiah K, Oliveira AO, Wong JS, Daub AC, Giorgini F, Muchowski P, Finkbeiner S. Deubiquitinase Usp12 functions noncatalytically to induce autophagy and confer neuroprotection in models of Huntington's disease. Nat Commun. 2018;9(1):3191. https://doi.org/10.1038/s41467-018-05653-z 
21. Chen X, Wang J, Gao X, Wu Y, Gu G, Shi M, Chai Y, Yue S, Zhang J. Tauroursodeoxycholic acid prevents ER stressinduced apoptosis and improves cerebral and vascular function in mice subjected to subarachnoid hemorrhage. Brain Res. 2020;1727:146566. https://doi.org/10.1016/j. brainres.2019.146566

22. Xu W, Gao L, Li T, Zheng J, Shao A, Zhang J. Apelin-13 alleviates early brain injury after subarachnoid hemorrhage via suppression of endoplasmic reticulum stress-mediated apoptosis and blood-brain barrier disruption: possible involvement of ATF6/CHOP pathway. Neuroscience. 2018;388:284-96. https://doi.org/10.1016/j. neuroscience.2018.07.023

23. Xu W, Li T, Gao L, Zheng J, Yan J, Zhang J, Shao A. Apelin-13/ APJ system attenuates early brain injury via suppression of endoplasmic reticulum stress-associated TXNIP/NLRP3 inflammasome activation and oxidative stress in a AMPKdependent manner after subarachnoid hemorrhage in rats. J Neuroinflammation. 2019;16(1):247.. https://doi. org/10.1186/s12974-019-1620-3

24. Zou R, Wang MH, Chen Y, Fan X, Yang B, Du J, Wang XB, Liu KX, Zhou J. Hydrogen-rich saline attenuates acute lung injury induced by limb ischemia/reperfusion via down-regulating chemerin and NLRP3 in rats. Shock. 2019;52(1):134-41. https://doi.org/10.1097/shk.0000000000001194

25. Ning K, Liu WW, Huang JL, Lu HT, Sun XJ. Effects of hydrogen on polarization of macrophages and microglia in a stroke model. Med Gas Res. 2018;8(4):154-9. https://doi. org/10.4103/2045-9912.248266

26. Kumagai K, Toyooka T, Takeuchi S, Otani N, Wada K, Tomiyama A, Mori K. Hydrogen gas inhalation improves delayed brain injury by alleviating early brain injury after experimental subarachnoid hemorrhage. Sci Rep. 2020;10(1):12319. https://doi.org/10.1038/s41598-02069028-5

27. Ohno $\mathrm{K}$, Ito $\mathrm{M}$, Ichihara $\mathrm{M}$, Ito $\mathrm{M}$. Molecular hydrogen as an emerging therapeutic medical gas for neurodegenerative and other diseases. Oxid Med Cell Longev. 2012;2012:353152. https://doi.org/10.1155/2012/353152

28. Takeuchi S, Mori K, Arimoto H, Fujii K, Nagatani K, Tomura S, Otani N, Osada H, Wada K. Effects of intravenous infusion of hydrogen-rich fluid combined with intra-cisternal infusion of magnesium sulfate in severe aneurysmal subarachnoid hemorrhage: study protocol for a randomized controlled trial. BMC Neurol. 2014;14:176. https://doi.org/10.1186/ s12883-014-0176-1

29. Afonso MB, Rodrigues PM, Simão AL, Ofengeim D, Carvalho T, Amaral JD, Gaspar MM, Cortez-Pinto H, Castro RE, Yuan $\mathrm{J}$, Rodrigues CM. Activation of necroptosis in human and experimental cholestasis. Cell Death Dis. 2016;7(9):e2390. https://doi.org/10.1038/cddis.2016.280

30. Chen J, Wang Y, Wu J, Yang J, Li M, Chen Q. The potential value of targeting ferroptosis in early brain injury after acute CNS disease. Front Mol Neurosci. 2020;13:110. https://doi.org/10.3389/fnmol.2020.00110
31. Chen J, Zhang C, Yan T, Yang L, Wang Y, Shi Z, Li M, Chen Q. Atorvastatin ameliorates early brain injury after subarachnoid hemorrhage via inhibition of pyroptosis and neuroinflammation. J Cell Physiol. 2021. https://doi. org/10.1002/jcp.30351

32. Zhuang Z, Zhou ML, You WC, Zhu L, Ma CY, Sun XJ, Shi JX. Hydrogen-rich saline alleviates early brain injury via reducing oxidative stress and brain edema following experimental subarachnoid hemorrhage in rabbits. BMC Neurosci. 2012;13:47. https://doi.org/10.1186/14712202-13-47

33. Feng Y, Wang R, Xu J, Sun J, Xu T, Gu Q, Wu X. Hydrogen-rich saline prevents early neurovascular dysfunction resulting from inhibition of oxidative stress in STZ-diabetic rats. Curr Eye Res. 2013;38(3):396-404. https://doi.org/10.3109/027 13683.2012.748919

34. Ohsawa I, Ishikawa M, Takahashi K, Watanabe M, Nishimaki K, Yamagata K, Katsura K, Katayama Y, Asoh S, Ohta S. Hydrogen acts as a therapeutic antioxidant by selectively reducing cytotoxic oxygen radicals. Nat Med. 2007;13(6):688-94. https://doi.org/10.1038/nm1577

35. Sugawara T, Ayer R, Jadhav V, Zhang JH. A new grading system evaluating bleeding scale in filament perforation subarachnoid hemorrhage rat model. J Neurosci Methods. 2008;167(2):327-34. https://doi.org/10.1016/j. jneumeth.2007.08.004

36. Li G, Dong Y, Liu D, Zou Z, Hao G, Gao X, Pan P, Liang G. NEK7 Coordinates rapid neuroinflammation after subarachnoid hemorrhage in mice. Front Neurol. 2020;11:551. https:// doi.org/10.3389/fneur.2020.00551

37. Schaible EV, Windschügl J, Bobkiewicz W, Kaburov $Y$, Dangel L, Krämer T, Huang C, Sebastiani A, Luh C, Werner C, Engelhard K, Thal SC, Schäfer MK. 2-Methoxyestradiol confers neuroprotection and inhibits a maladaptive HIF-1 $\alpha$ response after traumatic brain injury in mice. J Neurochem. 2014;129(6):940-54. https://doi. org/10.1111/jnc.12708

38. Das S, Chattopadhyay D, Chatterjee SK, Mondal SA, Majumdar SS, Mukhopadhyay S, Saha N, Velayutham R, Bhattacharya S, Mukherjee S. Increase in PPAR $\gamma$ inhibitory phosphorylation by Fetuin-A through the activation of Ras-MEK-ERK pathway causes insulin resistance. Biochim Biophys Acta Mol Basis Dis. 2021;1867(4):166050. https:// doi.org/10.1016/j.bbadis.2020.166050

39. Chen JH, Wu T, Xia WY, Shi ZH, Zhang CL, Chen L, Chen QX, Wang YH. An early neuroprotective effect of atorvastatin against subarachnoid hemorrhage. Neural Regen Res. 2020;15(10):1947-54. https://doi. org/10.4103/1673-5374.280326

40. Liu L, Xie K, Chen H, Dong X, Li Y, Yu Y, Wang G, Yu Y. Inhalation of hydrogen gas attenuates brain injury in mice with cecal ligation and puncture via inhibiting neuroinflammation, oxidative stress and neuronal apoptosis. Brain Res. 2014;1589:78-92. https://doi. org/10.1016/j.brainres.2014.09.030 
41. Choi KS, Kim HJ, DoSH, Hwang SJ, Yi HJ. Neuroprotective effects of hydrogen inhalation in an experimental rat intracerebral hemorrhage model. Brain Res Bull. 2018;142:122-8. https:// doi.org/10.1016/j.brainresbull.2018.07.006

42. Wang $P$, Zhao $M$, Chen $Z$, Wu G, Fujino $M$, Zhang $C$, Zhou W, Zhao M, Hirano SI, Li XK, Zhao L. Hydrogen gas attenuates hypoxic-ischemic brain injury via regulation of the MAPK/HO-1/PGC-1a pathway in neonatal rats. Oxid Med Cell Longev. 2020;2020:6978784. https://doi. org/10.1155/2020/6978784

43. Dohi K, Kraemer BC, Erickson MA, McMillan PJ, Kovac A, Flachbartova Z, Hansen KM, Shah GN, Sheibani N, Salameh T, Banks WA. Molecular hydrogen in drinking water protects against neurodegenerative changes induced by traumatic brain injury. PLoS One. 2014;9(9):e108034. https://doi. org/10.1371/journal.pone.0108034

44. Camara R, Matei N, Camara J, Enkhjargal B, Tang J, Zhang JH. Hydrogen gas therapy improves survival rate and neurological deficits in subarachnoid hemorrhage rats: a pilot study. Med Gas Res. 2019;9(2):74-9. https://doi.org/10.4103/2045-9912.260648

45. Yao L, Chen $H$, Wu Q, Xie K. Hydrogen-rich saline alleviates inflammation and apoptosis in myocardial I/R injury via PINK-mediated autophagy. Int J Mol Med. 2019;44(3):104862. https://doi.org/10.3892/ijmm.2019.4264

46. Chen H, Mao X, Meng X, Li Y, Feng J, Zhang L, Zhang Y, Wang Y, Yu Y, Xie K. Hydrogen alleviates mitochondrial dysfunction and organ damage via autophagy-mediated NLRP3 inflammasome inactivation in sepsis. Int J Mol Med. 2019;44(4):1309-24. https://doi.org/10.3892/ijmm.2019.4311

47. Wang Y, Wang L, Hu T, Wang F, Han Z, Yin Z, Ge X, Xie K, Lei $P$. Hydrogen improves cell viability partly through inhibition of autophagy and activation of PI3K/Akt/GSK3 $\beta$ signal pathway in a microvascular endothelial cell model of traumatic brain injury. Neurol Res. 2020;42(6):487-96. https://doi.org/10.1080/01616412.2020.1747717
48. Barancik M, Kura B, LeBaron TW, Bolli R, Buday J, Slezak J. Molecular and cellular mechanisms associated with effects of molecular hydrogen in cardiovascular and central nervous systems. Antioxidants (Basel). 2020;9(12). https:// doi.org/10.3390/antiox9121281

49. Zhang Z, Sun X, Wang K, Yu Y, Zhang L, Zhang K, $\mathrm{Gu} J$, Yuan X, Song G. Hydrogen-saturated saline mediated neuroprotection through autophagy via $\mathrm{PI} 3 \mathrm{~K} / \mathrm{AKT} / \mathrm{mTOR}$ pathway in early and medium stages of rotenone-induced Parkinson's disease rats. Brain Res Bull. 2021;172:1-13. https://doi.org/10.1016/j. brainresbull.2021.04.003

50. Ohta S. Direct targets and subsequent pathways for molecular hydrogen to exert multiple functions: focusing on interventions in radical reactions. Curr Pharm Des. 2021;27(5):595-609. https://doi.org/10.2174/138161282 6666200806101137

51. Yu Y, Yang $Y$, Yang $M$, Wang $C$, Xie K, Yu Y. Hydrogen gas reduces HMGB1 release in lung tissues of septic mice in an Nrf2/HO-1-dependent pathway. Int Immunopharmacol. 2019;69:11-8. https://doi. org/10.1016/j.intimp.2019.01.022

52. Chen H, Xie K, Han H, Li Y, Liu L, Yang T, Yu Y. Molecular hydrogen protects mice against polymicrobial sepsis by ameliorating endothelial dysfunction via an Nrf2/ HO-1 signaling pathway. Int Immunopharmacol. 2015;28(1):643-54. intimp.2015.07.034.

https://doi.org/10.1016/j.

53. Yu Y, Feng J, Lian N, Yang M, Xie K, Wang G, Wang $C, Y u$ Y. Hydrogen gas alleviates blood-brain barrier impairment and cognitive dysfunction of septic mice in an Nrf2-dependent pathway. Int Immunopharmacol. 2020;85:106585. https://doi.org/10.1016/j. 